\title{
Control of Speed and Power in a Humanoid Robot Arm Using Pneumatic Actuators for Human-Robot Coexisting Environment
}

\author{
Kiyoshi HOSHINO $^{\dagger a)}$, Member
}

SUMMARY A new type of humanoid robot arm which can coexist and be interactive with human beings are looked for. For the purpose of implementation of human smooth and fast movement to a pneumatic robot, the author used a humanoid robot arm with pneumatic agonist-antagonist actuators as endoskeletons which has control mechanism in the stiffness of each joint, and the controllability was experimentally discussed. Using Kitamori's method to experimentally decide the control gains and using I-PD controller, three joints of the humanoid robot arm were experimentally controlled. The damping control algorithm was also adopted to the wrist joint, to modify the speed in accordance with the power. The results showed that the controllability to step-wise input was less than one degree in error to follow the target angles, and the time constant was less than one second. The simultaneous input of command to three joints was brought about the overshoot of about ten percent increase in error. The humanoid robot arm can generate the calligraphic motions, moving quickly at some times but slowly at other times, or particularly softly on some occasions but stiffly on other occasions at high accuracy.

key words: pneumatic actuator, humanoid robot arm, simultaneous control of multiple joints, I-PD control, damping control

\section{Introduction}

Time has come when robots which coexist with human beings are looked for. The qualities requested of such robots are summarized into three different types: firstly, labor assisting work such as carrying of heavy objects, support to lying down and getting up, etc., secondly, work relating to hobbies and studies such as games and sports, making a companion of study, etc., and thirdly, household duties such as cleaning, putting things in order after meals, crime and disaster prevention, etc. Namely, only a robot helpful to our daily life can be a robot capable of coexisting with human beings. Such a robot is requested to make actions close to those of a human being, i.e. move quickly at some times but slowly at other times, or particularly softly on some occasions but stiffly on other occasions.

But a robot is greatly different in size and weight from the human, because many of the conventional robots use a motor as actuator [1]-[6] under the present conditions. There is a crucial request for a new type of actuator which is lightweight, powerful and excellent in safety and environmental protection, for the human cooperative and coexistence environments.

Manuscript received September 11, 2007.

Manuscript revised December 9, 2007.

${ }^{\dagger}$ The author is with the Graduate School of Systems and Information Engineering, University of Tsukuba, Tsukuba-shi, 3058573 Japan.

a)E-mail: hoshino@esys.tsukuba.ac.jp

DOI: 10.1093/ietisy/e91-d.6.1693
As a new actuator solving such problems, studies have been being made on pneumatic actuator [7], [8]. A pneumatic actuator is available in various forms such as air cylinder, pneumatic artificial muscle, rubber actuator, etc. and studies are being made in a variety of fields such as humanoid robot, medical services and welfare. And it is a good tool to investigate the computational brain science with [9]. But it is difficult to make the actuators work as desired [10], for example, with classical PID control, because of unstable output characteristics.

Air cylinder seems to be useful as actuator for robot because it has a lot of advantages such as compact size and light weight, simple structure and easy maintenance, generation of large power, etc. compared with motor. However, the conventional piston structure had a great defect that it is difficult to generate the performance of immobilizing the piston at a given position, as stiffness which is inversely proportional to the mechanical impedance. For that reason, the authors' group developed a piston structure capable of producing stiffness by providing the air cylinder with a valve which well regulates inflow of air into the cylinder and outflow from the cylinder, and realized a lightweight humanoid robot arm having the same size and degrees of freedom as the humans, the cylinder shouldering the agonistantagonist muscle structure, by using the cylinder itself as endoskeleton [11].

The purpose of the present study is to propose a new type of humanoid robot arm which can coexist and be interactive with human beings. The arm has seven degrees of freedom with air cylinder actuators, but it can move quickly at some times but slowly at other times, or particularly softly on some occasions but stiffly on other occasions at high accuracy, so as to generate the calligraphic writing motions.

\section{System Construction}

\subsection{Air Cylinder Actuator with Controllable Stiffness}

In this chapter, the author describe an outline of the pneumatic robot arm with changeable stiffness at each joint [11] developed by the authors' research group. We paid attention to the fact that, if you either stop entry of air into a cylinder or outflow from the cylinder or reduce the flow rate of that air, a passive reaction serving as resistance to the piston motion is produced, with the repulsion of the compressed air or the flow rate resistance of the air. We utilized the reaction as stiffness of the air cylinder. This led us to devise an 


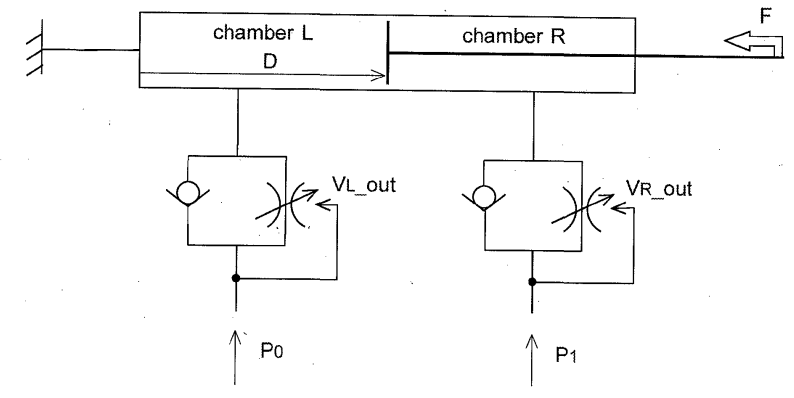

Fig. 1 Auto choke valve.

automatic choke valve as shown in Fig. $1 . \mathrm{P}_{0}$ and $\mathrm{P}_{1}$ indicate the target pressures in each chamber respectively. Firstly, we disposed, in the respective air channels, a valve which makes outflow of air difficult, and provided it with a function of automatically reducing the sectional area of flow rate based on the pressure of that air. This is intended to improve efficiency by linking increase of air pressure for pushing the piston in the direction of reversing the piston motion with the act of making it difficult for that high-pressure air to escape. Still more, because a large volume of high-pressure air must flow into the cylinder, to make the piston move at high speed, we also installed a unidirectional valve as bypass, to enable free inflow of air. With the functioning of this automatic choke valve, we realized design of a cylinder having stiffness.

\subsection{Robot Arm with Endoskeleton Actuators}

A cylinder with large diameter and stroke is required to obtain a large output with air pressure. It is difficult to incorporate such cylinder in a humanoid robot arm the size and movable range of which are of a level same as that of the humans and, in addition, such cylinder occupies the greater part of the space in the arm, having negative influences on the arm motion, etc. For that reason, it has been a normal practice, with conventional humanoid robots, to take the method of providing a drive mechanism composed of a cylinder placed on the outside of the arm, to transmit motive power to the hand tip by means of a wire and pulley mechanism. However, such method requires a large space for the drive mechanism, increasing the overall weight of the mechanism and inevitably causing a drop of accuracy of motion and efficiency due to elongation or friction of the wire.

For that reason, we devised to utilize cylinder as endoskeleton, by incorporating an air cylinder on the shaft of the upper arm joint and the forearm joint of a humanoid robot arm, by making use of high strength of the air cylinder itself. Moreover, by providing two valves on one cylinder as shown in Fig. 1, we also realized to furnish the robot arm with muscle structure or agonist-antagonist muscle structure producing arm motions of the human [11]. And, we realized slewing motions as well, by means of a torsion mechanism which converts linear motions of the air cylinder concerned

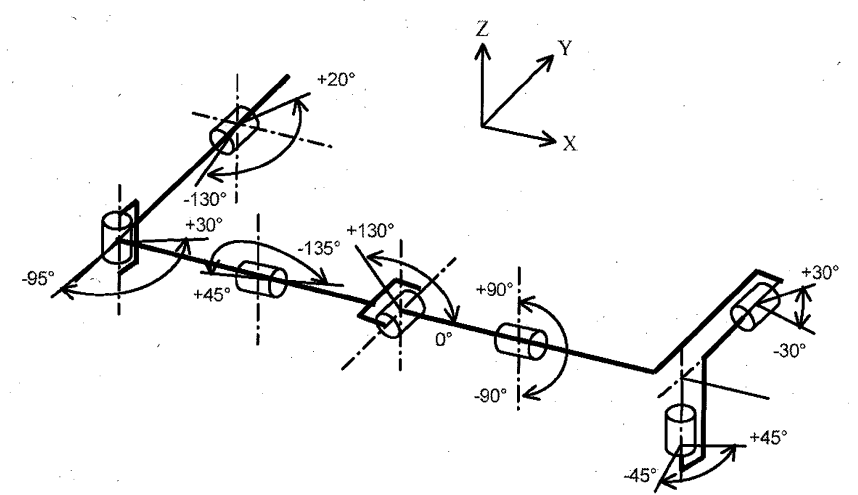

Fig. 2 Movable range of each joint.

into rotational motions around the shaft. Because of this endoskeleton structure, we realized a lightweight robot arm, in a size about equal to that of the human, having seven degrees of freedom and a wide movable range as shown in Fig. 2.

\section{Control Algorithm}

\section{1, Modeling}

The human upper limbs have seven degrees of freedom in total, i.e. three degrees of freedom at shoulder joint, two degrees of freedom at elbow joint, and two degrees of freedom at wrist joint. It is the three degrees of freedom, namely flexion-extension and adduction-abduction of shoulder and flexion-extension of elbow, that increase the movable range in the three-dimensional orbit produced by the hand. Therefore, we attempted to control and investigate those three joints in the present study.

In controlling those three joints of the humanoid robot arm, we first led out equations of motion based on the Lagrangian method. Since the coordinate system changes with displacement and translation of joint angle, the respective simultaneous coordinate transformation matrices given as $T_{0}$ and $T_{1}$ may be expressed with the following equations:

$$
\begin{aligned}
T_{0} & =\left[\begin{array}{cccc}
\cos \theta_{1} & -\sin \theta_{1} & 0 & 0 \\
\sin \theta_{1} & \cos \theta_{1} & 0 & 0 \\
0 & 0 & 1 & 0 \\
0 & 0 & 0 & 1
\end{array}\right] \\
T_{1} & =\left[\begin{array}{cccc}
\cos \theta_{2} & 0 & \sin \theta_{2} & L_{1} \\
0 & 1 & 0 & 0 \\
-\sin \theta_{2} & 0 & \cos \theta_{2} & 0 \\
0 & 0 & 0 & 1
\end{array}\right]
\end{aligned}
$$

where, $\theta_{1}$ is the flexion-extension angle of shoulder, $\theta_{2}$ is the adduction-abduction angle of shoulder, $\theta_{3}$ is the flexionextension angle of elbow, and $L_{1}$ is the length of link from shoulder to elbow. By multiplying them with transformation matrix each time when the coordinate is transformed, one may determine the respective local coordinates as world coordinate.

Lagrangian equation is used for determining 
Lagrangian function from kinetic energy and potential energy of the entire link, and for leading out an equation of motion mechanically by substituting that function in the Lagrangian equation. The equation of motion of respective joints can be expressed with the following equation:

$$
J \theta^{\prime \prime}(t)+D \theta^{\prime}(t)+h\left(\theta^{\prime \prime}(t), \theta^{\prime}(t)\right)+g(\theta(t))=\tau(t)
$$

where, $J$ is the moment of inertia, $D$ is a coefficient of viscous friction, $h$ is a non-linear term, $g$ is a gravity term, and $\tau$ is torque.

However, because PID control is handled only in linearity, approximation for linearization is required. Therefore, in the present study, we added non-linear term and gravity term as torque for non-linear compensation, and linear approximated Eq. (3) as in Eq. (4).

$$
\begin{aligned}
& J \theta^{\prime \prime}(t)+D \theta^{\prime}(t)=\tau^{\prime}(t) \\
& \tau^{\prime}(t)=h\left(\theta^{\prime}(t), \theta^{\prime \prime}(t)\right)+g(\theta(t))+\tau(t)
\end{aligned}
$$

\subsection{I-PD Control}

Much of robot arm control is made with PID control. In the present study, we used I-PD control. I-PD control is a kind of PID preceded by proportional and derivative gains, and its control algorithm can be expressed with the following equation:

$$
u(t)=-K p y(t)+K i \int_{0}^{t} e(\tau) d \tau-K d \frac{d y(t)}{d t}
$$

where, $u(t)$ is the amount of operation, $y(t)$ is the amount of control, $e(\tau)$ is a deviation, $K p$ is a proportional gain, $K i$ is an integral gain, and $K d$ is a derivative gain.

In I-PD control, changes in the target value are not reflected immediately in the amount of operation, unlike in general PID control, because the deviation concerns only the integral gain. For that reason, overshoot is controlled even with a large change in the target value. However, it also presents a problem of slower response. It becomes important therefore how to determine an optimal gain without drop of response.

\subsection{Estimation of Gain}

\subsubsection{Identification of Parameters}

If you use, in the estimation of gain, a method of determining an optimal value from the behavior or value observed in actual motions, a large burden is imposed on the arm because there is no way to foresee the motion of the robot arm. For that reason, in the present study, we made estimation by using Kitamori's method [12], [13] which enables to theoretically lead out an estimated value.

An equation of motion of a linear approximated robot arm was obtained as shown in Eq. (4). Considering an angle control, the transfer function $G(s)$ can be expressed with the following equation because the amount of control is $\theta(t)$, and the amount of operation is $\tau(t)$.

$$
G(s)=\frac{b}{s(s+a)}, \quad a=\frac{D}{J}, \quad b=\frac{1}{J}
$$

where, $J$ is the moment of inertia, and $D$ is a coefficient of viscous friction.

The parameters $a=D / J$ and $b=1 / J$ included in Eq. (7) are unknown parameters unmeasurable with a measuring instrument. For that reason, we identified those parameters through experiments. Since Eq. (8) is obtained by putting as $y(t)=\theta^{\prime}(t)$ in Eq. (5), the transfer function $G^{\prime}(s)$ turns into Eq. (9) of linear system. Moreover, Eq. (9) turns into Eq. (10) when expressed in the standard form of linear system.

$$
\begin{aligned}
& J y^{\prime}(t)+D y(t)=\tau(t) \\
& G^{\prime}(s)=\frac{b}{s+a}, \quad a=\frac{D}{J}, \quad b=\frac{1}{J} \\
& G^{\prime}(s)=\frac{K}{1+T s}, \quad T=\frac{1}{a}, \quad K=\frac{b}{a}
\end{aligned}
$$

The unknown parameters $a$ and $b$ are determined as in the following equations, if the time constant $T$ and the gain $K$ are determined:

$$
a=\frac{1}{T}, \quad b=K a=\frac{K}{T}
$$

\subsubsection{Estimation of I-PD Control Gain by Kitamori's Method}

Kitamori's method, also called partial model matching method, is a method which consists in designing parameters in a way to make a closed-loop transfer function $T(s)$ agree with or come close to a desired transfer function called model.

Equation (6) turns into Eq. (12) with Laplacian transformation. From Eqs. (9) and (12), the closed-loop transfer function $T(s)$ turns into Eq. (13) of cubic system. To make Eq. (13) completely agree with the model Eq. (14), all you have to do is to select gain as in Eq. (15). However, since $\omega$ is a parameter regarding quick response, and $\alpha_{1}, \alpha_{2}$ are parameters regarding attenuation respectively, it is often the case that Butterworth standard form, binomial standard form, or ITAE minimum standard form are used as attenuation parameters. In the present study, we used the binomial standard form $\alpha_{1}=3, \alpha_{2}=3$. The value of $\omega$, for which there is no fixed method of determination, was determined by the method of try and error through experiments.

$$
\begin{aligned}
& U(s)=-K p \Theta(s)+\frac{K i}{s} \Theta(s)-K d s \Theta(s) \\
& T(s)=\frac{b K i}{s^{3}+(a+b K d) s^{2}+b K p s+b K i} \\
& T_{M}(s)=\frac{\omega^{3}}{s^{3}+\alpha_{1} \omega s^{2}+\alpha_{2} \omega^{2} s+\omega^{3}} \\
& K i=\frac{\omega^{3}}{b}, \quad K p=\frac{\alpha_{2} \omega^{2}}{b}, \quad K d=\frac{\alpha_{1} \omega-a}{b}
\end{aligned}
$$




\subsection{Control of Power of Wrist Joint}

With a PID preceded by proportional and differential gains only, it happens on some rare occasions that the tip of the pen gets off the paper face at some moment. To prevent such problem, we loaded a strain gauge on the portion grasping the pen, to make perform a simple power control using the wrist joint only in the vertical direction. In the present study, we adopted damping control which consists in modifying the speed in accordance with the power generated in the robot. The control algorithm is expressed with the following formula:

$$
v=v_{0}+A p
$$

where, $v_{0}$ is an upper limit set speed, $v$ is an effective speed, $p$ is a drag, and $A$ is an admittance matrix. Admittance matrix $A$ is a coefficient which performs mapping from the upper limit set speed $v_{0}$ and the effective speed $v$. If the tip of the pen is not in contact with the paper face, $v=v_{0}$ is established, from drag $p=0$, and the tip of the pen pushes the paper face more strongly. On the contrary, in the case where the tip of the pen is in contact with the paper face and remains stationary in the vertical direction, the effective speed $v$ becomes equal to zero $(v=0)$ and, by substituting it in Eq. (16), you obtain $p=v_{0} / A$. Therefore, the robot continues pressing the pen on the paper face until the detected power $p$ becomes equal to $v_{0} / A\left(p=v_{0} / A\right)$, enabling to draw stable hair-pen characters.

\section{Experiments}

\subsection{Method}

We made calculations by Kitamori's method, by using parameters obtained through identification of parameters, and estimated gains experimentally while changing responsive parameters. At that time, parameters with good response not causing any large overshoot were adopted as optimal parameters.

\subsection{Results and Discussions}

\subsubsection{Results of Control of Respective Joints}

For experiments of motion, we first set a state in which the respective joints come to zero degree as initial position, and made control toward target angles.

Figure 3 shows examples of responses in adduction and abduction of shoulder. In the movable range of 40 to $60^{\circ}$, we could control the motions with an overshoot of $2^{\circ}$ or so and an error against target angle smaller than $1^{\circ}$ (Fig. 3 (a)). Figure $3(\mathrm{~b})$ shows an example of response in a motion made by changing the target angle. A motion of passing to the target angle could also be controlled with smooth motions with an overshoot of $2^{\circ}$ or so.

Figure 4 shows examples of responses in adduction

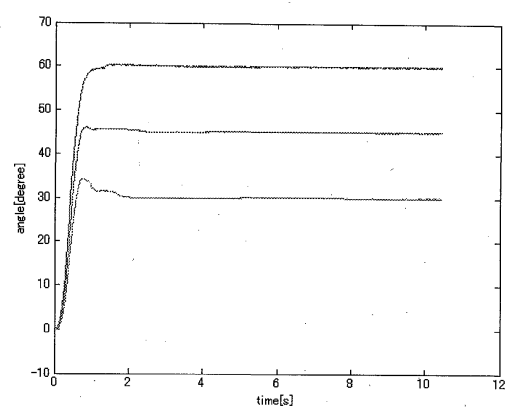

(a)

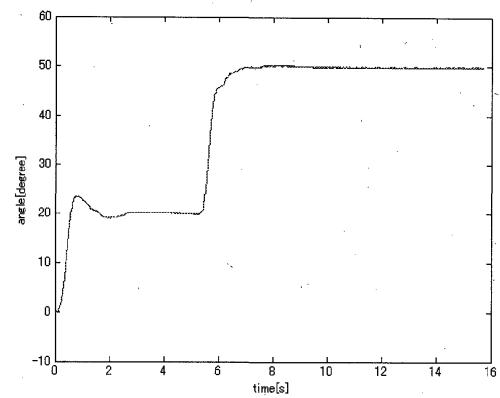

(b)

Fig. 3 Examples of responses in shoulder's adduction and abduction.

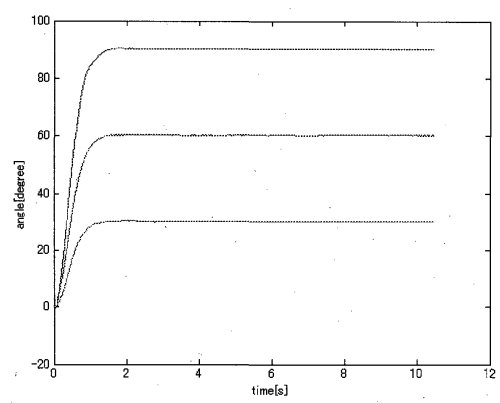

(a)

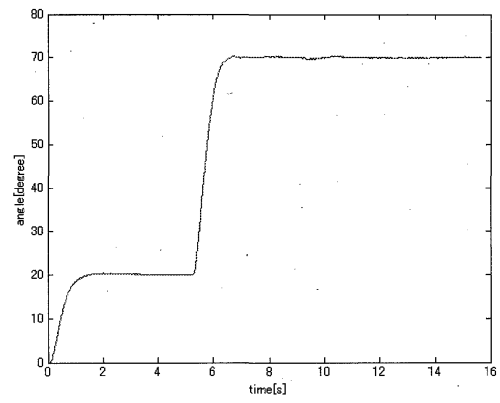

(b)

Fig. 4 Examples of responses in elbow's flexion and extension.

and abduction of elbow. In adduction and abduction of elbow (Fig. 4 (a)), we could control the overshoot under $1^{\circ}$ up to $90^{\circ}$, and control the error against target angle under $1^{\circ}$ thereafter. Figure 4(b) shows an example of response in a motion made by changing the target angle. In this case too, a motion of passing to the target angle could be controlled with smooth motions with an overshoot of $1^{\circ}$ or so. 


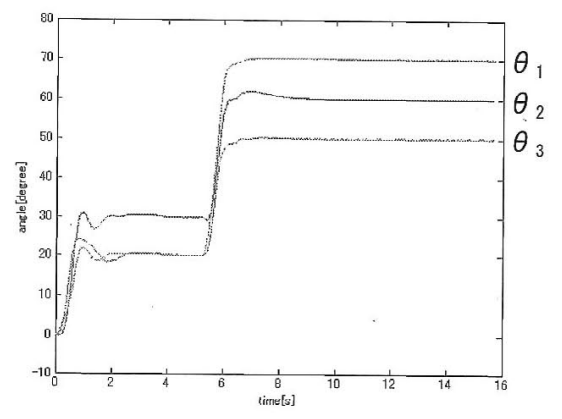

Fig. 5 An example of three joints responses to simultancous step-wise input. $\theta_{1}, \theta_{2}, \theta_{3}$ indicate flexion and extension of shoulder, adduction and abduction of shoulder, flexion and extension of elbow, respectively.

\subsubsection{Results of Control at Three Joints}

Since good results were obtained in the time response of respective joints in the preceding section, we conducted simultaneous control of three joints in succession.

Figure 5 indicates the results of an experiment on how a change of target angle is followed. In the same way as in the case where the respective joints were moved one by one, we changed the target angle in steps as $0^{\circ} \rightarrow 30^{\circ} \rightarrow 60^{\circ}$ for flexion and extension of shoulder, as $0^{\circ} \rightarrow 20^{\circ} \rightarrow 50^{\circ}$ for adduction and abduction of shoulder, and as $0^{\circ} \rightarrow 20^{\circ} \rightarrow$ $70^{\circ}$ for flexion and extension of elbow. For each joint, there are cases where an overshoot of approximately $10 \%$ against the target angle is produced, but they are controlled without vibrations, with an error under $1^{\circ}$ against the target angle.

As was described in the first chapter, the air is easily compressed and expanding. The irregularity therefore arises at the speed and the precise adjustment is difficult to be done in the pneumatic actuator. But, on the contrary, the air can be supplied everywhere and spent by returning to the atmosphere. Our system supplies chambers with the large air by fully opening the inlets, and carries out the high-speed and high-precise control by closing and opening the outlets. The system which uses abundant volume of air can obtain the high responsibility even though comparatively simple controller where gain decision is also easy like I-PD is adopted.

\subsubsection{Control of Wrist Joint}

Figure 6 indicates examples of calligraphy by pneumatic robot arm with seven degrees of freedom. Figure 7 shows the snapshots in which a robot arm is writing the Chinese character "牛肉 (beef)" by using a hair pen. The starting, end, and via points of the lines in the character were beforehand given to the system. The more a line was curved, the more via points were used. The coordinates of the via points were heuristically selected so that the line will be naturally interpolated. The plain paper of A4 size was used. We can see that a quick and accurate writing motion is realized, in spite of the fact that this robot arm is a multipurpose robot arm adopting a pneumatic actuator. With robots [14], [15] for which a motor is used as actuator, it was necessary to

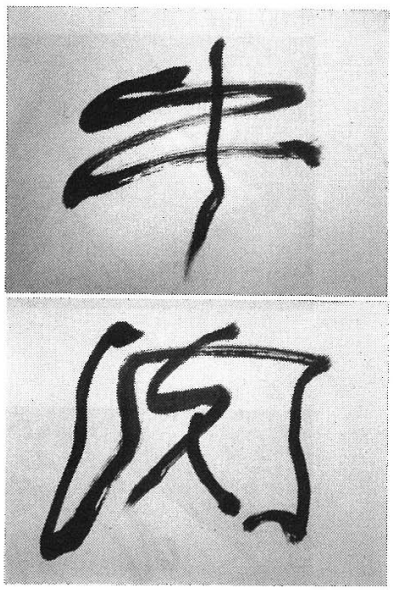

Fig. 6 Examples of calligraphy by pneumatic robot arm.

trace the object material such as paper face, etc. carefully by means of a writing tool, because of an essentially large rigidity, thus requiring a long working time for writing characters. On the other hand, with the robot arm of the present study which is of pneumatic system and is therefore essentially soft, there is no need of providing any particular control algorithm such as compliance control, etc. As for the rapidity of actions, it achieves a time about equal to that of writing motion of a human being [16] or even a shorter time required. The author may say it shows that a pneumatic actuator is performing quick and soft actions, while maintaining accuracy.

In a writing motion, a proper pressing force of the tip of the pen to the paper face is required. If the pressing force is too small, the tip of the pen may get off the paper face easily at some moment. If, on the contrary, that force is too large, there may be cases where the paper is broken. Damping control adopted in the present study is a control algorithm for modifying the speed of a manipulator, etc. according to the drag acting on the object material. And, the amount of that modification of speed is determined by admittance matrix. The amount of the admittance matrix element strongly affects the drag acting on the object material at the time of execution of work. For example, in the case of a small value of matrix element, the positioning error is not absorbed, producing an excessive drag. On the contrary, in the case of a large matrix element value, the control system becomes unstable and, as a result, some divergent drag may act in some cases. As described above, the behaviors of a manipulator are greatly influenced by power control parameters, and it is important to set the parameters properly. However, a power control algorithm has a lot of parts which cannot be designed analytically or which are difficult to do so. For that reason, a method has been proposed which consists in determining control parameters through learning [17].

Moreover, the main purpose of damping control is, firstly, to prevent any excessive force acting on the object material and, secondly, to absorb the positioning error of the object material. However, it is reported that, in a conditional 

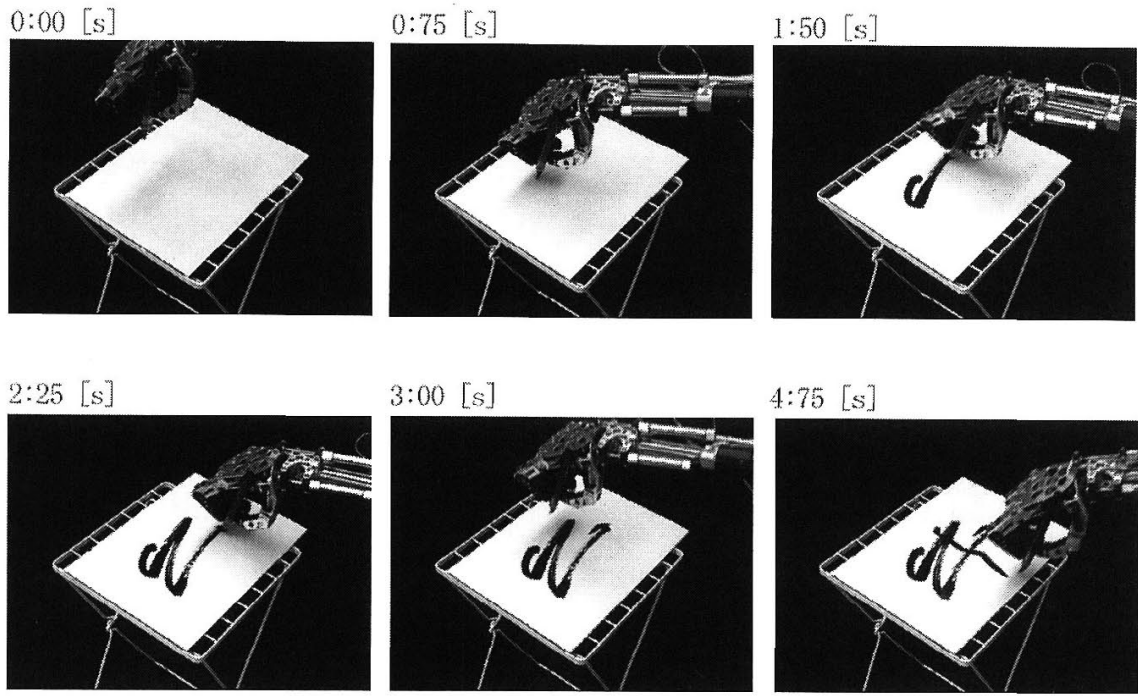

$3: 00[\mathrm{~s}]$

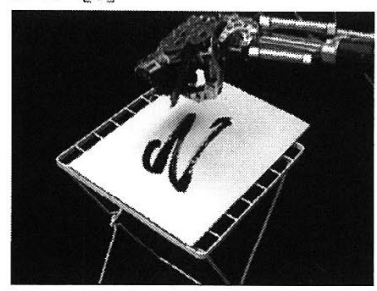

$4: 75[\mathrm{~s}]$

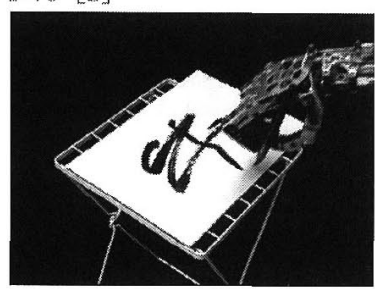

Fig. 7 Snapshots of arm movement.

formula for admittance matrix element, it is impossible to determine the amount of matrix element, because this formula is a homogenous linear inequality regarding matrix element [18].

However, air has compression and expansion properties, as mentioned before. Such properties are unfit for accurate positioning or generation of rigidity. On the other hand, however, they are fit for preventing any excessive force acting on the object material. In any case, if it is possible to realize a comparatively accurate positioning control, by means of a pneumatic actuator having properties of preventing any excessive force acting on the object material, we may expect realization of a proper power control, even if the amount of admittance matrix element is not determined rigorously. As a result of experiments, no accidental separation of the tip of the pen from the paper face took place at all, even in writing motions made by combining the damping control algorithm with a PID preceded by proportional \& differential gains. This fact shows that, even without learning of power control parameters through repeated attempts or analytical parameters design, it is possible to realize desired motions, by means of an air cylinder actuator which enables accurate positioning and rigidity adjustment by reducing the degree of opening of exhaust properly depending on the target air pressure.

\section{Conclusion}

Robots which coexist and be interactive with human beings are looked for. That is to say, these robots should move quickly at some times but slowly at other times, or particularly softly on some occasions but stiffly on other occasions at high accuracy. Pneumatic actuators fit with the purpose, but the studies on pneumatic actuators and the control algorithms, control with conventional pneumatic actuator is rather difficult to make because of instability of output characteristics.
In the present study, therefore, the author carried out the experiments of simultaneous control of three joints by experimentally determining the gain with Kitamori's method and with the use of I-PD for the controller. The humanoid robot developed by our group was used, where a pneumatic control valve capable of generating stiffness and the cylinder itself constitute the endoskeleton and the respective joints have an agonist-antagonist muscle structure.

In the experiments, while an overshoot of approximately $10 \%$ against the target angle was produced, with simultaneous of joints, under the influence of their respective motions, they could be controlled with an error under $1^{\circ}$ about follow-up to the target angle and with a time constant less than one second. Quick and smooth motions as in the humans could be realized, in a pneumatically driven robot arm for which accurate and rapid positional control is said to be difficult.

The author also adopted damping control algorithm to control the power of wrist joint, to modify the speed in accordance with the power. The humanoid robot arm with seven degrees of freedom generates the calligraphic motions, moving quickly at some times but slowly at other times, or particularly softly on some occasions but stiffly on other occasions at high accuracy.

\section{References}

[1] K. Hoshino and I. Kawabuchi, "Pinching at finger tips for humanoid robot hand," J. Robotics and Mechatronics, vol.17, no.6, pp.655-663, 2005.

[2] D. Shiokata, A. Namiki, and M. Iwata, "Robot dribbling using a high-speed multifingered hand and a high-speed vision system," 2005 IEEE/RSJ Intl. Conf. Intelligent Robots and Systems, pp.2097-2102, 2005.

[3] J. Butterfass, M. Fischer, M. Grebenstein, S. Haidacher, and G. Hirzinger, "Design and experiences with DLR hand II," World Automation Congress, vol.15, pp.105-110, 2004.

[4] H. Kawasaki, T. Komatsu, and K. Uchiyama, "Dexterous anthropomorphic robot hand with distributed tactile sensor: Gifu hand II," 
IEEE/ASME Trans. Mechatronics, vol.7, pp.296-303, 2002.

[5] C.S. Lovchic and M.A. Diftler, "A dexterous robot hand for space," Proc. 1999 IEEE Intl. Conf. Robotics and Automation, pp.907-912, 1999.

[6] K.J. Kyriakopoulos, J.V. Riper, A. Zink, and H.E. Stephanou, "Kinematics analysis and position/force control of the anthrobot dexterous hand," IEEE Trans. Systems, vol.27, no.1, pp.95-103, 1997.

[7] S.C. Jacobsen, J.E. Wood, D.F. Knutti, and K.B. Biggers, "The UTAH/M.I.T Dextrous Hand: Work in progress," Int. J. Robot. Res., vol.3, no.4, pp.21-50, 1984.

[8] Shadow Dextrous Hand, http://www.shadow.org.uk/products/ newhand.shtml

[9] M. Katayama and M. Kawato, "Learning trajcctory and force control of an artificial muscle arm by parallel-hierarchical neural network model," in Advances in Neural Information Processing Systems, 3, ed. R.P. Lippmann, J.E. Moody, and D.S. Touretzky, pp.436-442, Morgan Kaufmann, 1991.

[10] D. Sasaki, T. Noritsugu, and M. Takaiwa, "Development of pneumatic soft hand for human friendly robot," J. Robotics and Mechatronics, vol.15, no.2, pp.164-171, 2003.

[11] K. Hoshino and I. Kawabuchi, "Mechanism of humanoid robot arm with 7 DOFs having pneumatic actuators," IEICE Trans. Fundamentals, vol.E89-A, no.11, pp.3290-3297, Nov. 2006.

[12] S. Shin and T. Kitamori, "Model reference learning control for discrete-time nonlinear systems," Adaptive Systems in Control and Signal Processing 1989, pp.101-106, Pergamon Press, 1990.

[13] S. Shin and T. Kitamori, "Variable robust adaptive law," Proc. Workshop on Robust Control, pp.179-185, Springer-Verlag, 1992.

[14] http://matsuda.c.u-tokyo.ac.jp/sci/project/lab-ikeuchi/text_7.php

[15] http://www.com.rd.pref.gifu.jp/ ceram/topics/GCI023.pdf

[16] http://www.shosha.kokugo.juen.ac.jp/oshiki/jyugyo/bridge1/jitsugi/ bridge 1 jitsugi.html

[17] S. Hirai, T. Inatsugi, and K. Iwata, "Learning of admittance matrix elements for manipulative operations," Proc. IEEE/RSJ Intl. Conf. Intelligent Robots and Systems, vol.2, pp.763-768, 1996.

[18] S. Hirai and K. Iwata, "Derivation of damping control parameters based on geometric model," Proc. IEEE Intl. Conf. Robotics and Automation, vol.2, pp.87-92, 1993.

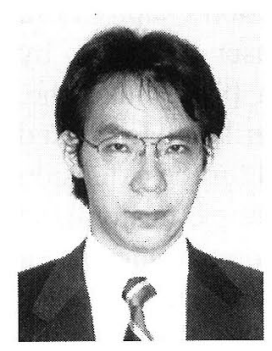

Kiyoshi Hoshino received two doctor's degrees; one in Medical Science in 1993, and the other in Engineering in 1996, from the University of Tokyo respectively. From 1993 to 1995 he was an assistant professor of Tokyo Medical and Dental University School of Medicine. From 1995 he became an associate professor of Biological Cybernetics Lab, University of the Ryukyus. He is now working as an associate professor of University of Tsukuba. From 1998 to 2001 he was jointly appointed as a senior researcher of PRESTO "Information and Human Activity" project, Japan Science and Technology Agency (JST). From 2002 to 2005 he was a project leader of SORST project of JST. His research interests include computational brain science and humanoid robot design. He is a member of RSJ and JSMBE. 